\title{
Identification of ERD using Fuzzy Inference Systems for Brain-Computer Interface
}

\author{
I. Dzitac, T. Vesselényi, R.C. Ţarcă
}

\author{
Ioan Dzitac \\ Aurel Vlaicu University of Arad \\ Department of Mathematics-Informatics \\ Elena Dragoi St., 2, Complex M \\ Arad, Romania \\ E-mail:ioan.dzitac@uav.ro
}

Tiberiu Vesselényi, Radu Cătălin Ţarcă

University of Oradea

Faculty of Management and Technological Engineering

Universitatii St. 1, 410087

Oradea, Romania

E-mail:tvesselenyi@yahoo.co.uk,rtarca@uoradea.ro

\begin{abstract}
A Brain-Computer Interface uses measurements of scalp electric potential (electroencephalography - EEG) reflecting brain activity, to communicate with external devices. Recent developments in electronics and computer sciences have enabled applications that may help users with disabilities and also to develop new types of Human Machine Interfaces. By producing modifications in their brain potential activity, the users can perform control of different devices. In order to perform actions, this EEG signals must be processed with proper algorithms. Our approach is based on a fuzzy inference system used to produce sharp control states from noisy EEG data.
\end{abstract}

Keywords: Event Related Desynchronization (ERD), Brain-Computer Interface (BCI), electroencephalography (EEG), fuzzy inference system.

\section{Introduction}

Devices that are using brain (cortical or scalp) electric potentials which allow brain function signals to control machines (or computers) are called brain-computer interfaces (BCI). It had been shown that BCI systems using steady state visual evoked potentials have allowed healthy subjects to communicate with computers [1].

Non-invasive, electroencephalogram (EEG)-based BCI technologies can be used to control a limb prothesis, for the use of a computer program, and for other functions such as environment control or entertainment. BCI technologies can improve the lives of people with neurological disorders and also can restore motor control by guiding activity-dependent brain plasticity [2]. A review of the advantages of BCIs which shows that they can provide even a good candidate for space applications is presented in [3]. Some BCI implementations are using sensory-motor rhythms as it is presented in [4]. The interest shown for BCI technology is motivated by the applicability for helping disabled, for gaming, and as a tool in cognitive neuroscience [5]. In [6] a model is proposed which includes a set of definitions of the typical entities encountered in a $\mathrm{BCI}$, diagrams which explain the structural correlations among them and a detailed description of the timing of a trial.

In this paper we present some of our original experiments with BCI, and EEG data processing methods applied. 


\section{BCI applications}

Important application for BCIs can be found in the medical field. A good example for this is presented in [7] where BCI methodology based on self-regulation of slow-cortical potentials of the EEG is used. This kind of two choice task is called "brain-switch" [8], but there are other applications in which multiple choices are employed.

Another important application of BCI is represented by cognitive experiments during which the researchers try to better understand some sequences of brain functions. In [9] for example the researchers use information derived from previous EEG recordings to inform the analysis of functional magnetic resonance imaging (fMRI) data collected for the same behavioral task.

Robotics and environment control applications are also emerging in the studies of this area. For example in [10] a study was made in order to determine the most discriminative features for a BCI system based on statistically significant differences between two energy density maps calculated from EEG signals during two different motor tasks. In [11] it is shown that simple rehabilitation tasks can be performed and household robotic devices can be easily controlled by means of BCI technology. Robotic arms, which have been controlled by means of cortical invasive interfaces in animal studies, could be the next frontier for noninvasive BCI applications. A review of current robotic technologies that are relevant to BCI is presented in [12]. As it can be seen from the references in this field, employment of prothesis and rehabilitation is a largely studied area in the field of BCI applications [13], [14], [15]. Several other applications of BCI are designed for entertainment, gamming and learning to concentrate or to achieve a certain desired mental state.

\section{Considerations on EEG signals and signal processing methods used in BCI}

Most of the BCI devices developed by now are based on the electric potentials of scalp known as EEG, which is noninvasive opposing to those based on cortical potentials where the electrodes are in direct contact with the cortex (electrocorticography - ECoG). A special method is using EEG signals combined with an appropriate model of volume conduction and of neuro-electrical sources (high-resolution electroencephalography) [16].

In order to intentionally generate the electrical potentials of the brain two kinds of tasks are widely used which are also correlated with different regions of the brain: the sensory-motor (or sometimes motor imagery) tasks and those tasks which imply some kind of processing called mental or cognitive-related tasks (i.e. simple syntactic analysis of words). The sensory-motor tasks are by far mostly used because their direct implication in limb movement [17], [4], [18]. Examples of mental or cognitive-related task studies are given in [19] and [20].

In sensory-motor tasks an often used method is to employ Event-related desynchronization (ERD) which is a reduction in EEG signal amplitude in a specific frequency band $(8-12 \mathrm{~Hz})$ as a result of a particular event. When a subject is making a hand movement, or even just imagine it, a reduction in sensory-motor signal amplitude can be observed in certain parts of the scalp. In order to use this in a BCI application the proper components of the signal must be found. The signals measured by sensors, particularly those used in noninvasive techniques, are known to be very noisy due to a series of potentials generated by other biological or artificial sources than brain potentials. One of the major challenges of BCI devices is to filter out the unwanted components of the signals. Here both hardware and software solutions are to be applied. The other major task is to uniquely recognize a specific pattern in the useful component of the signals which is meant to trigger a specific command. The number of sensors placed in specific areas 
of the scalp can vary from 1 to 36 which are read in on different channels of the device. Each of these channels can be filtered separately to monitor a specific, larger or narrower, frequency domain.

Researchers applied a wide range of techniques to filter, preprocess and analyze signals and to produce decisions.

Statistical Approach. In [21] the researchers studied spike sorting by introducing a mathematical model consisting of three Gaussian waveforms, which appropriately represents the general shapes of action potentials. Then they searched for the best-fit waveform for each noise-corrupted spike based on the model, using peak fitting method. The performance of the proposed method was assessed with synthesized neural recordings composed by spike templates and white Gaussian noise in various signal to noise ratio environments. Gaussian process (GP) classification to binary discrimination of motor imagery EEG data is described in [22]. A robust Bayesian linear regression algorithm is presented in [23] that automatically detects relevant features and excludes irrelevant ones, all in a computationally efficient manner.

Spectral Methods. In [24] the researchers investigated spectral power changes both in intracranial ECoG recordings in epilepsy patients and in non-invasive EEG recordings optimized for detecting high gamma band (a specific frequency domain in EEG signals) activity in healthy subjects. In order to characterize the non-Gaussian information contained within the EEG signals, a new feature extraction method based on "bispectrum" is proposed in [25] and applied to the classification of right and left motor imagery.

In [26] a novel method is presented for detecting frequency-frequency coupling between the electrical output of cortical areas as measured by ECoG, electroencephalography EEG and magnetoencephalography MEG, the biphase-locking value. The method is specifically sensitive to non-linear interactions, i.e. quadratic phase coupling across frequencies. This method was employed to study signals measured from pre-motor area, in the motor cortex.

Wavelet Methods. Wavelet transforms are applied in [27] to study motor imagery tasks. In this work the continuous wavelet transform (CWT) was applied together with Student's twosample t-statistics for 2D time-scale feature extraction, where features are extracted from EEG signals recorded from subjects performing left and right motor imagery. In [28] a novel method was proposed for the feature extraction of electroencephalogram (EEG) based on wavelet packet decomposition (WPD). In [29] the assessment of wavelet transform (WT) as a feature extraction method was used in representing the electrophysiological signals.

Genetic Algorithms. Positive potentials at a latency of about $300 \mathrm{~ms}$ in EEG (P300) are largely used to study brain functions. This pattern is used as sign of cognitive function in decision making processes. In [30] P300 detection approach based on some features and a statistical classifier was implemented. The optimal feature set was selected using a genetic algorithm from a primary feature set including some morphological, frequency and wavelet features and was used for the classification of the data.

Artificial Neural Networks and Support Vector Machines. In [31] the authors investigated if an artificial neural network-based model for closed-loop-controlled neural prostheses could use neuromuscular activation recorded from individuals with impaired spinal cord to predict their end-point gait parameters (such as stride length and step width). This ANN-based model allows a seamless incorporation of neuromuscular activity, detected from paralyzed individuals, to adaptively predict their altered gait patterns, which can be employed to provide closed-loop feedback information for neural prostheses. Support vector machine implementations are used in [32], [33], [34]. The results indicate that the proposed algorithms are promising for future use of rehabilitative BCI applications in neurologically impaired patients.

Fuzzy logic. In [35] a new method is introduced, which combines information from different classic time series similarity measures, using a fuzzy fusion framework. This method is accurate 
and reliable in P300 detection. This framework is used to combine two computationally simple signal detection methods: "peak picking" and "template matching". Fusion takes place in the last step (decision-making step) by means of a fuzzy rule-base. In order to predict the gripping force from the EEG signals in [36] a methodology that uses subsequent signal processing methods is used: filtering, principal component analysis, and the phase-demodulation method. A fuzzy inference system is then used to predict the gripping force from the processed EEG data. Neuro-fuzzy methods are also to be considered as a highly efficient way to deal with uncertain information.

The paper [37] introduces a number of modifications to the learning algorithm of the selforganizing fuzzy neural network (SOFNN) to improve computational efficiency. An analysis of the SOFNN effectiveness when applied in an electroencephalogram (EEG)-based brain-computer interface (BCI) involving the neural-time-series-prediction-preprocessing (NTSPP) framework is also presented, where a sensitivity analysis of the SOFNN hyper-parameters was performed using EEG data recorded from three subjects during left/right-motor-imagery-based BCI experiments. The aim of this analysis was to eliminate the need to choose subject and signal specific parameters for EEG preprocessing. The results indicate that a general set of NTSPP parameters chosen provide the best results when tested in a BCI system.

A new brain-computer interface design using fuzzy ARTMAP (FA) neural network, as well as an application of the design is proposed in [38]. The objective of this BCI-FA design is to classify the best three of the five available mental tasks for each subject using spectral amplitude values of electroencephalogram (EEG) signals. The findings show that the average BCI-FA outputs gave very low error using the best triplets of mental tasks identified from the classification performances of FA. In [39] a subject-based feature extraction method using the fuzzy wavelet packet in brain-computer interfaces (BCIs) is presented. In [40] is presented a new method introduced in [34], which combines information from different classic time series similarity measures, using a simple fuzzy fusion framework. This method is accurate and reliable in P300 (a positive event-related component occurring $300 \mathrm{~ms}$ after stimulus onset) detection. Fusion takes place in the decision-making step by means of a fuzzy rule-base. Compared to similar works on electroencephalogram-based (EEG-based) BCI datasets, in spite of being computationally simple, this new technique's performance is comparable to very complicated methods. In [41] an analyze of electroencephalogram (EEG) signals of imaginary left and right hand movements, an application of brain-computer interface (BCI) is presented. The researchers propose here to use an adaptive neuron- fuzzy inference system (ANFIS) as the classification algorithm. ANFIS has an advantage over many classification algorithms in that it provides a set of parameters and linguistic rules that can be useful in interpreting the relationship between extracted features. The continuous wavelet transform is used to extract highly representative features from selected scales.

\section{Method and results}

The 10-14 Hz frequency band of EEG signals is, among others, associated with those cortical areas that are most directly connected to the brain's normal motor output channels. Movement or preparation for movement is typically accompanied by a decrease of amplitude in this band over sensory - motor cortex. This decrease has been labeled "event-related desynchronization" or ERD [42]. Opposite to ERD, amplitude increase, or "event-related synchronization" (ERS) occurs in the post-movement period and with relaxation. Furthermore, and most relevant for BCI applications, ERD and ERS occur also with motor imagery (i.e., imagined movement) and they do not require actual movement.

In this research our goal is to analyze EEG signals by observing ERD and ERS characteristics 
and find a suitable algorithm to automatically detect these characteristics by using a fuzzy inference system. We consider that given the high noisiness of EEG signals a fuzzy or neurofuzzy approach would be desirable.

In our experiments subjects were asked to perform several hand movements at irregular times (with random intervals between two movements) during a session of 40 seconds. The hand movement consists of pushing a switch button. The subjects were also asked to concentrate on the action (hand movement) imagining it before. The EEG signals collected from a single electrode placed in C3 point on the scalp were registered. Electrode placement location is shown in figure 2. The switch on-off states were also registered using a separate acquisition device which is triggered to start data acquisition at the same time as the EEG signal acquisition, so the EEG signals and the switch on - off states data covers the same time period.

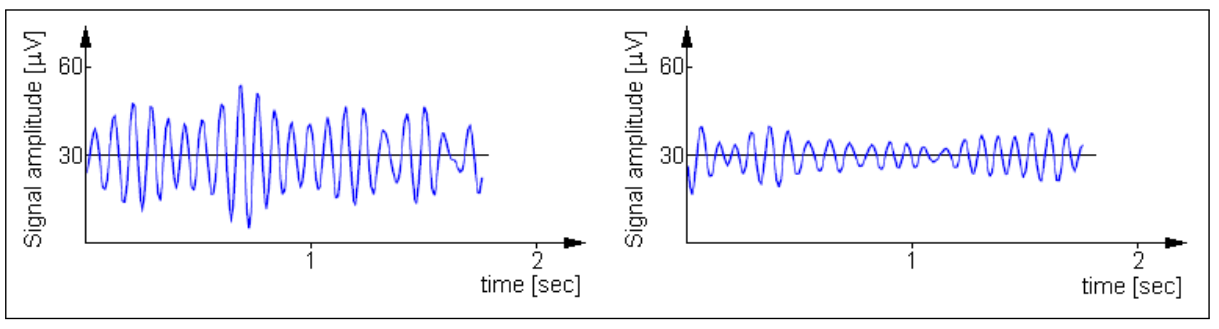

Figure 1: Theoretical EEG signals in $12 \mathrm{~Hz}$ frequency band. a) resting hand; b) moving hand (amplitude decrease - ERD).

A total of five healthy male adults (aged 25-48) participated in this study. None of the subjects had participated in the same experiments before and the subjects had a short basic training for the task. The subjects were informed about the nature and purpose of the experiments and consented to participate.

EEG signals were acquired using a BST112 8-channel amplifier from VEB MESSGERATEWERK ZVONIZ and for A/D conversion and transmission of data to a PC, a National Instruments NI-USB 6251 DAQ board had been used. The accuracy of the measuring system is of $1.5259 \mu \mathrm{V}$. The $\mathrm{Ag}-\mathrm{AgCl}$ electrode was placed in the $\mathrm{C} 3$ point on the scalp, using a conductive gel. As voltage reference, the A1 point was used (figure 2) and the ground was located on the right leg. Data were sampled at a sample rate of $1000 \mathrm{~Hz}$ and were transmitted through the USB port to a $\mathrm{PC}$ for storage and processing. The data acquisition and processing programs were written in MATLAB language.

Every subject has made 3 training sessions to accommodate with the environment and the equipment and then 4 sessions of 40 seconds each were recorded.

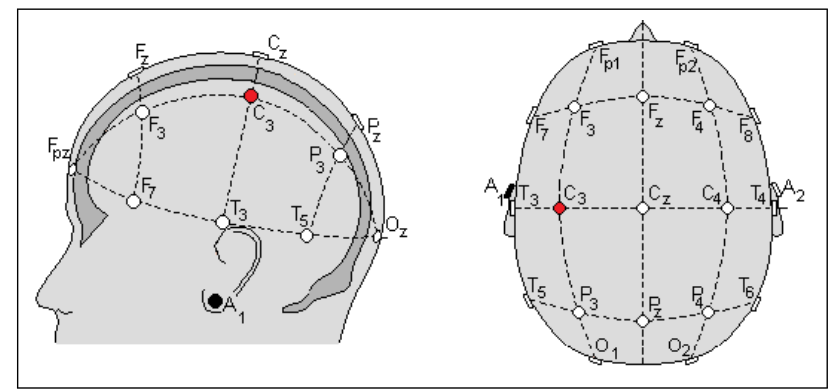

Figure 2: Location of electrode on the scalp in point C3.

The diagram of a sample acquisitioned signal is given in figure 3. As it can be observed the signal is affected by low level noise resulting in a variable offset across it's whole length. 


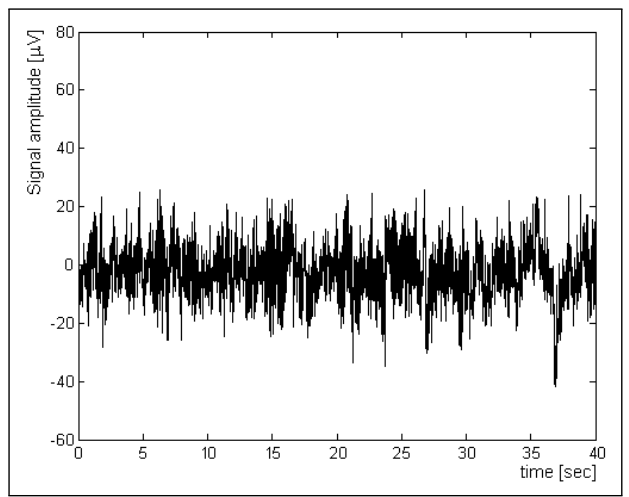

Figure 3: A sample of acquisitioned EEG signal.

This can be eliminated by using a running average filter which gives local average values which then are extracted from the original signal (sEEG1). If $n$ is the length of the signal $\mathrm{v}$ (in samples) and $\mathrm{m}$ is the running average filter length given by the vector $\mathrm{u}$, then the length of the average will be $\mathrm{m}+\mathrm{n}-1$. The running average $\mathrm{w}$ is given by convolution of the filter vector with the signal as it is shown in formulae 1 :

$$
w(k)=\sum_{j} u(j) \cdot v(k-j+1)
$$

where $\mathrm{k}$ is the index of the resulting signal $\mathrm{w}$ and $\mathrm{j}$ is the index of the filter $\mathrm{u}$. In this case all values of $\mathrm{u}$ are equal to $1 / \mathrm{m}$. In this case we used $\mathrm{m}=200$, which for a sampling rate of 1 $\mathrm{kS} / \mathrm{sec}$ will not generate errors for the studied frequency band (centered on $12 \mathrm{~Hz}$ ).

The running average values are shown in figure 4 (red). According to the convolution theorem, that convolution of signal and the digital filter's impulse response is nearly the same as multiplying their Fourier transforms. This can be used to speed up the calculations if it can be shown that the results are accurate up to an acceptable limit.

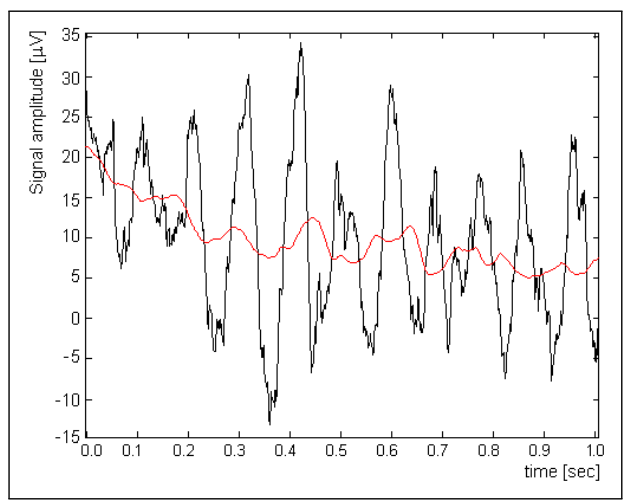

Figure 4: Detail of the acquisitioned signal for a 1 second time span (black). Running average values of the signal (red).

After extraction of the running average the resulting signal (sEEG2), is free of low frequency noises as it can be seen in the diagram given in figure 5 .

A further enhancement of the signal can be obtained by reducing the random peaks, employing a running average filter of the same form as before, but this time keeping the resulting average values. This has the effect of smoothing the signal without modifying the studied components. 


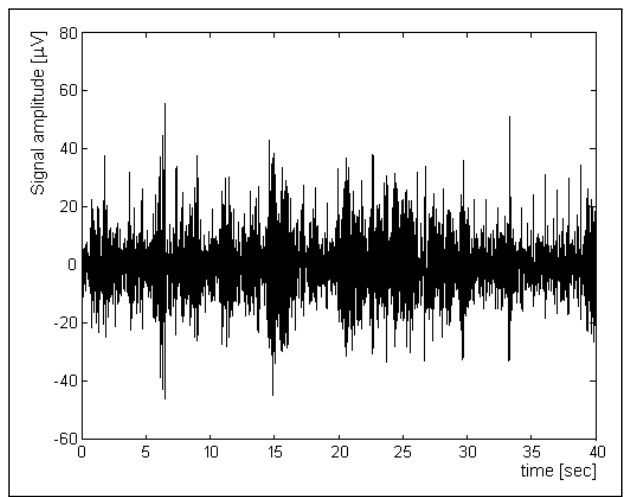

Figure 5: The signal given in figure 3, with running average values extracted.

This time we used $m=20$. The resulting signal (sEEG3) is shown in detail for a time span of 1 second in figure 6. Studying this signal we can observe that the dominating frequency is around 12-14 $\mathrm{Hz}$ but still having some low frequency components.

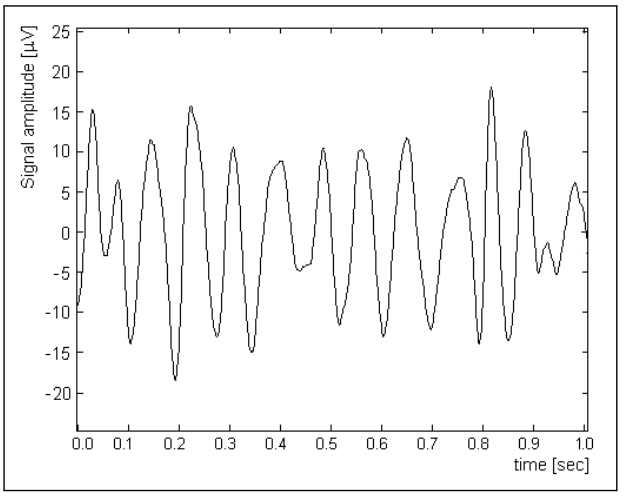

Figure 6: Detail of processed signal given in figure 5, for a time span of 1 second.

In order to further analyze the signal we have to switch to frequency domain analysis because time domain diagrams can no longer be enhanced. In case of frequency domain analysis of acquisitioned digital signals spectral amplitudes are computed using the Fast Fourier Transform (FFT - which is a variant of the Fourier transform used in numeric computing). A FFT amplitude value is given by formulae 2 :

$$
A(k)=\sum_{j=1}^{N} a(j) \omega_{N}^{(j-1)(k-1)}
$$

where: $\omega_{N}=e^{\frac{(-2 \pi \cdot i)}{N}}, \mathrm{~N}$ - number of samples; a(j) - amplitude of $\mathrm{j}$ - th sample in the signal.

In order to obtain the required spectrum we will use only the real part of the obtained FFT amplitudes $(\operatorname{real}(\mathrm{A}(\mathrm{k})))$.

As we want to analyze changes in the signal the FFT algorithm should be applied not on the entire length of the signal but with a suitably sized window which is moved step by step on the whole length of the signal. In our case we used a window of size NFFT $=256$. The number of partitions is NP = integer(m/NFFT). Some samples at the end of the signal will be lost but these can be only a quarter of a second which in our case is reasonable (given a 40 seconds long signal). 
Representing the result we will obtain a diagram of FFT amplitudes as a function of time and frequency as it is shown in figure 7 . The results had been interpolated using a cubic spline interpolation method in order to smooth the borders between partitions.

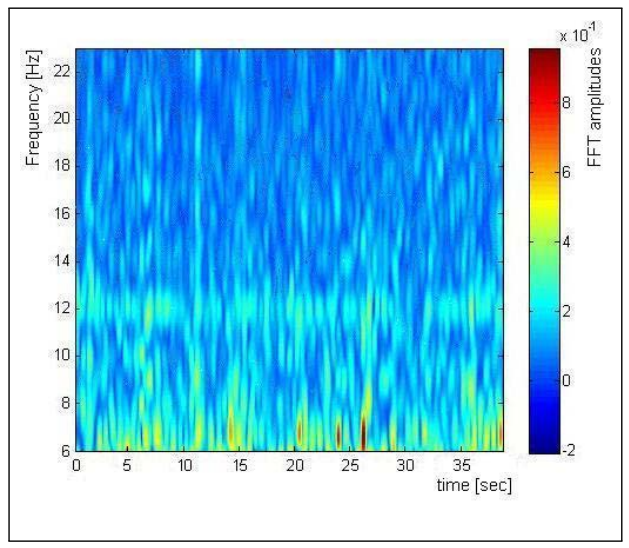

Figure 7: Time frequency diagram of the processed signal. The frequency band centered on 12 $\mathrm{Hz}$ is clearly observable.

If we select a slice of this diagram at a specific moment in time we ca obtain the spectra of the signal. Such spectra are given in figures 8 (resting hand) and 9 (moving hand).

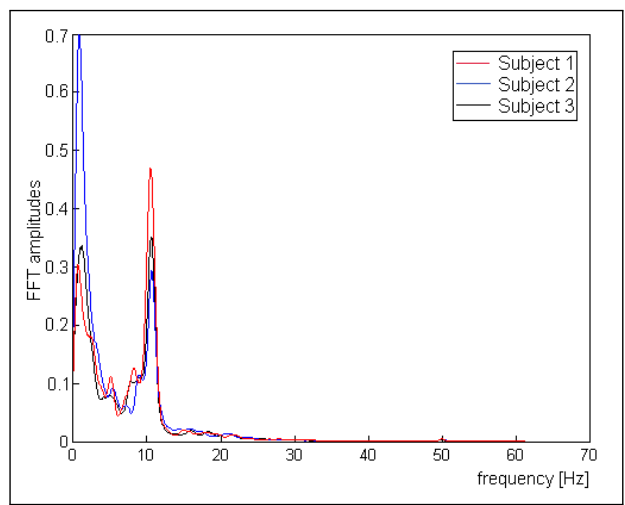

Figure 8: FFT amplitudes of the EEG signal for resting hand.

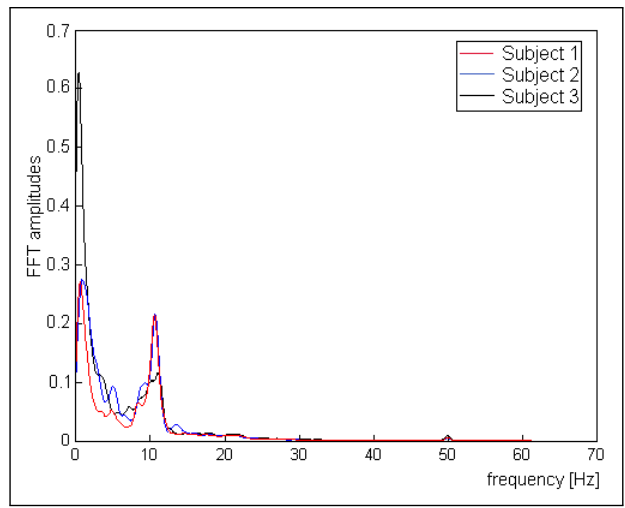

Figure 9: FFT amplitudes of the EEG signal for moving hand.

If we represent a slice of the time-frequency diagram for a specific frequency we will obtain 
the variation in time of FFT amplitude at that frequency. As ERD and ERS are occurring in a frequency band of $10-14 \mathrm{~Hz}$ choosing a center frequency of $12 \mathrm{~Hz}$ the resulting diagram is shown in figure 10 (black). On this diagram we had superimposed the switch on-off states (red). We can observe that the "ON" states (which are showing the real movement of the hand) mainly corresponds to the drop of signal amplitude of the chosen frequency. This should show the occurrence of ERD. Also there can be observed a rise of the curve corresponding with time intervals when the switch is not activated (the subject is relaxed). This should show the occurrence of ERS. In case of ERD the anticipation of the real movement, the fact that the amplitude is dropping earlier than the hand movement occurs shows that it happens due to motor imagery.

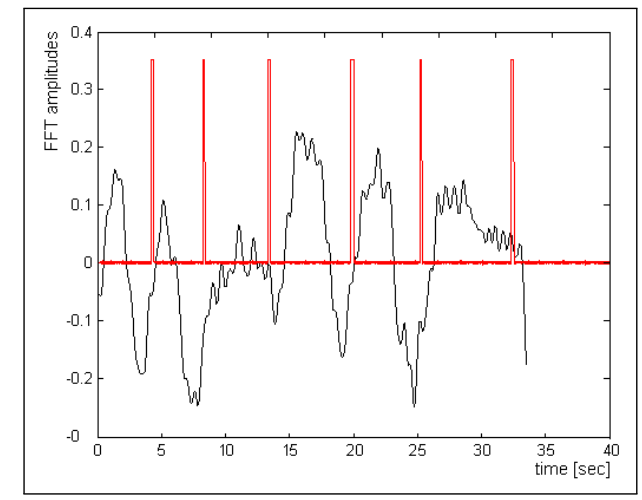

Figure 10: FFT amplitude versus time for the processed signal at the frequency of $12 \mathrm{~Hz}$ (black) and switch on-off states diagram (red).

As it can be seen in figure 10, and it had been observed in almost all of the studied cases, these characteristics can not be expressed with simple parameters (i.e. value of amplitude or the slope of the amplitude drop). Hence, in order to automatically detect ERD occurrence in FFT amplitude signals some fuzzy or neuro-fuzzy algorithms can be applied which may be capable of recognizing these kinds of amplitude drops. Fuzzy Logic is widely used in modelling and identification problems and in applications where data sets are obtained with a greater or smaller degree of uncertainty [43], [44], [45].

In the followings we will use an adaptive neuro-fuzzy inference system (ANFIS) to solve this issue. ANFIS is implemented in the Fuzzy Logic Toolbox of the MATLAB environment and uses a hybrid learning algorithm in order to identify parameters of Sugeno-type fuzzy inference systems [46]. It applies a combination of the least-squares method and the backpropagation gradient descent method for training membership function parameters to emulate the given training data set. The data sets obtained and processed, as it has been described, will be used for training and checking of the ANFIS system. After training, this system can be used to identify portions of the EEG signal which meet the ERD characteristics.

From the acquisitioned and processed data we had chosen 14 intervals which represents true ERD characteristics and which we will use to train, check and validate the fuzzy identification algorithm. In order to apply the algorithm the amplitudes must be normalized and the time intervals must be scaled so all the datasets representing ERD characteristics will have the same amplitude domain and the same number of samples. For normalization the datasets were divided with the maximum value of each dataset and for time scaling a linear interpolation algorithm had been used.

Two of the datasets were chosen as input data for training and checking. These datasets are shown in figure 11. Another dataset had been chosen as output dataset shown in figure 12 . 


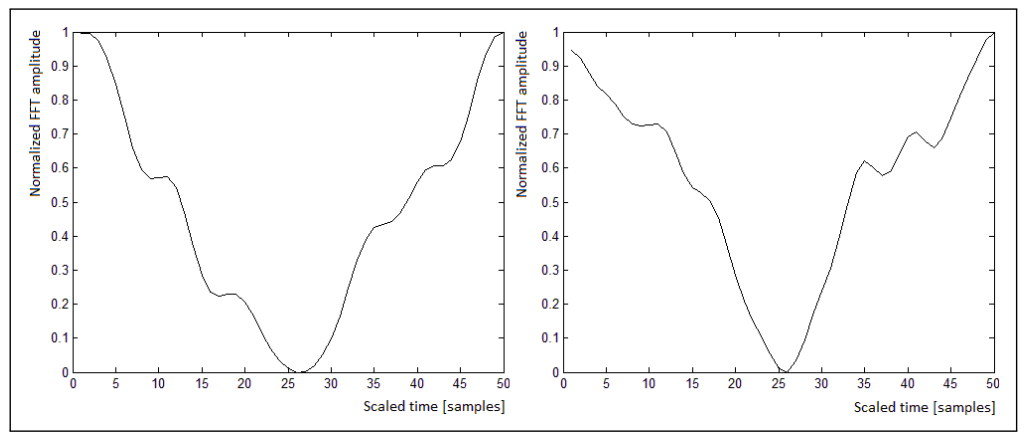

Figure 11: Normalized FFT amplitudes versus scaled time for two ERD characteristics; a. training input, b. checking input.

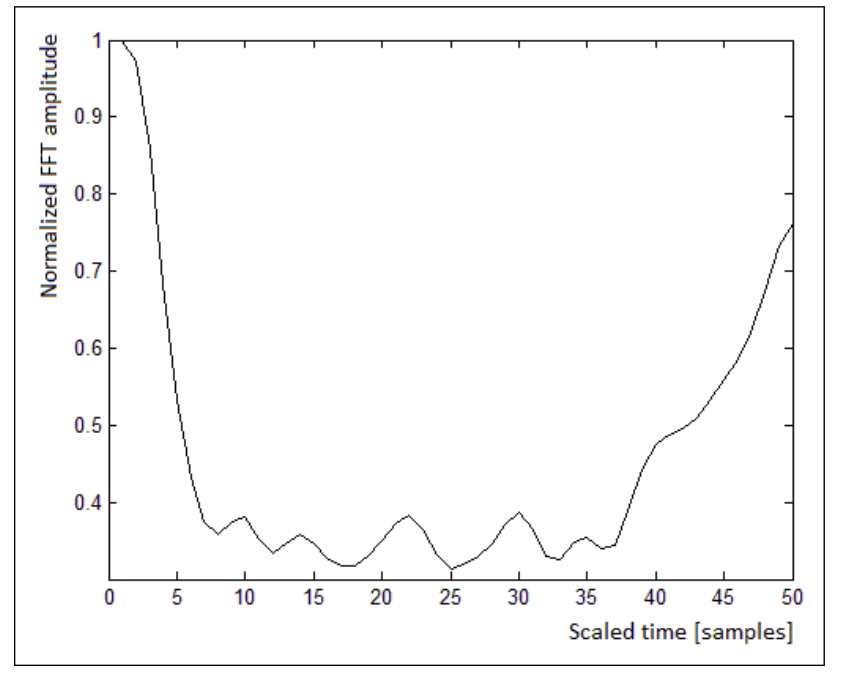

Figure 12: Normalized FFT amplitudes versus scaled time, output dataset.

In the training process the initial membership functions are modified to fit the training input - output dataset and in the checking process these membership functions are tuned in correspondence with the checking input-output datasets. The diagrams of the membership functions obtained after training are given in figure 13.a. and after tuning in figure 13.b.

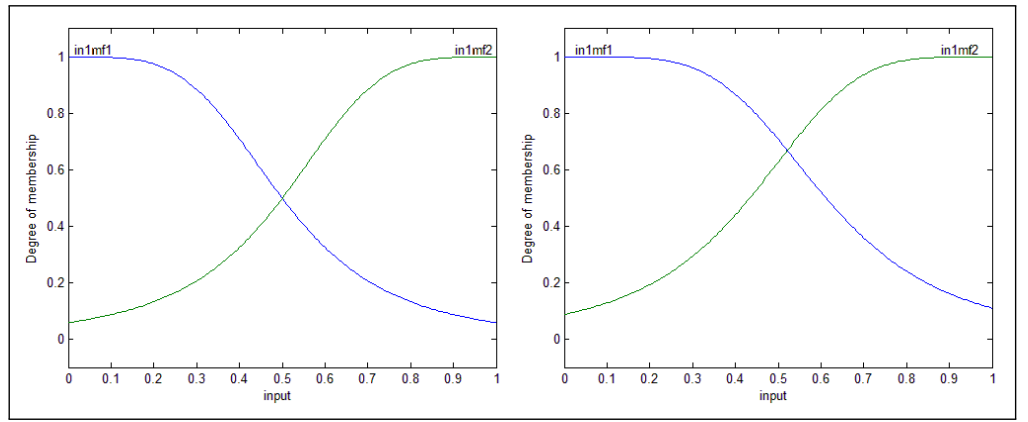

Figure 13: Membership functions obtained after training (a.) and after tuning (b.).

The fuzzy inference system parameters are: number of nodes: 12; number of linear parameters: 4; number of nonlinear parameters: 6 ; number of fuzzy rules: 2 .

Once the fuzzy inference system was trained and tuned we used 14 ERD characteristic 
datasets to verify their outputs (VO) compared to the results obtained for the dataset used as output in the training process (TO) which is used as reference. System output of the two different validation datasets are given as examples in diagrams of figure 14 .

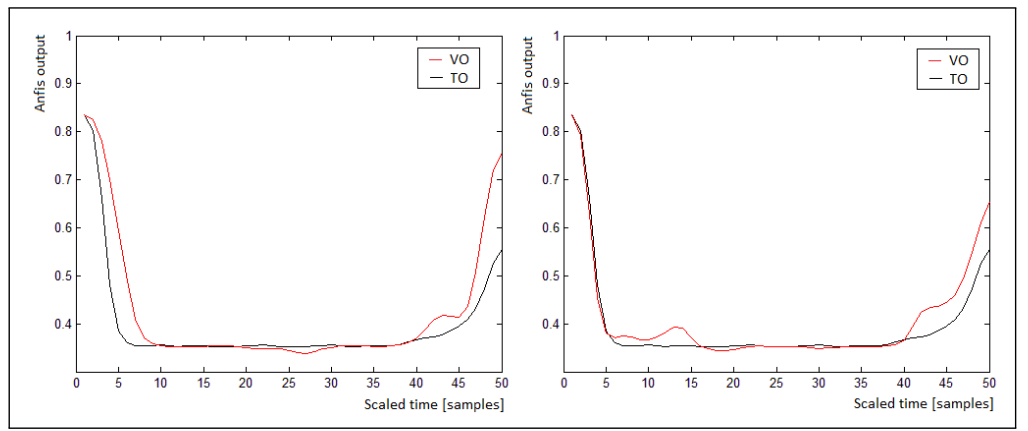

Figure 14: Validation and training system output data diagrams.

For each validation dataset the root mean square (RMS) values of the difference between VO and TO datasets were computed. These values are shown in the diagram in figure 15.

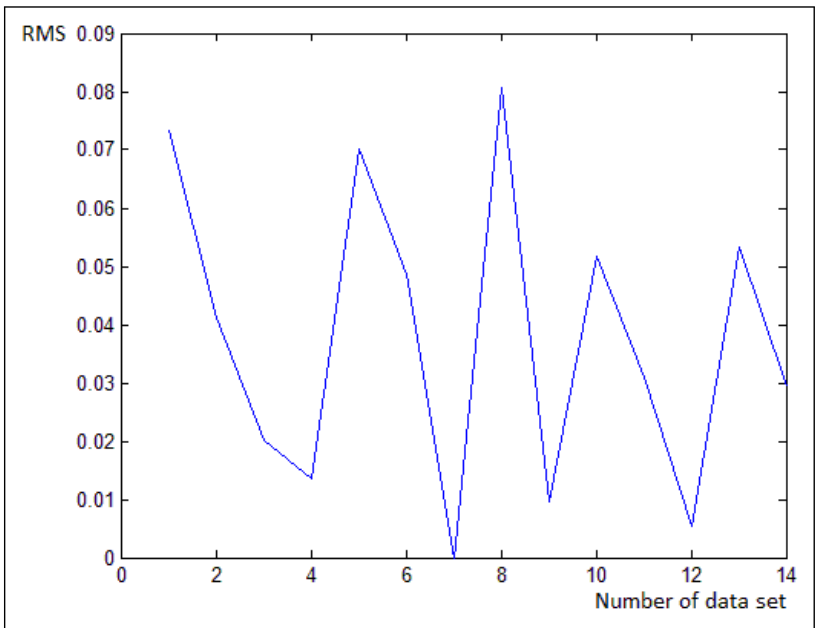

Figure 15: RMS values of difference between VO and TO for 14 validation datasets.

In order to identify if a dataset is a true ERD component or not the RMS values of difference between VO and TO can be used as a classifier. On the basis of RMS values span obtained for known true ERD components we can distinguish ERD components in real time acquired signals using simple or complex threshold techniques.

\section{Conclusions and future works}

In this work we described our experiments and the development of data processing for EEG signals, focusing on identification of ERD characteristics which can be used in brain - computer interfaces. The experiments showed that EEG signals had to be carefully acquired and processed in order to identify ERD components. Data acquisition equipment quality, careful selection of subjects and subject focus capacity are of major importance in achieving successful results. Data preparation had been done considering simple and time effective algorithms which can be used for real time processing of EEG signals. 
Further studies must be made to validate the described fuzzy inference system on a larger number of datasets for both true and false ERD components in order to establish it's robustness. There is still in discussion whether to use output in the training process dataset as reference for ERD identification or to find another more appropriate reference. In a future work we will try to answer these questions.

\section{Acknowledgements}

We thank our colleagues Simona Dzitac, Cristina Hora and Ioan Moga for her/his practical contributions in this research.

Also we thank the professors Hariton Costin and Horia-Nicolai Teodorescu for the positive and constructive comments and recommendations on our manuscript, which significantly contributed to improving the quality of this paper.

We are very honored to thank professor Lotfi A. Zadeh for his generous openness in collaborations with Romanian researchers and for his visits in Romanian cities Arad (2003: He was granted as Doctor Honoris Causa of Aurel Vlaicu University of Arad) and Oradea (2008: As Keynote Speaker in our conference ICCCC 2008 and Exploratory Workshop on Natural Language Computation- EWNLC 2008).

Dear professor Zadeh, celebrating the 90th birthday, we wish you a good health, long life, and new interesting achievements!

\section{Bibliography}

[1] Brendan Z. Allison, Dennis J. McFarland, Gerwin Schalk, Shi Dong Zheng, Melody Moore Jackson, Jonathan R. Wolpaw, Towards an independent brain-computer interface using steady state visual evoked potentials, Clinical Neurophysiology, 119(2): 399-408, 2008

[2] Janis J Daly, Jonathan R., Wolpaw, Brain-computer interfaces in neurological rehabilitation, The Lancet Neurology, 7(11): 1032-1043, 2008

[3] Carlo Menon, Cristina de Negueruela, José del R. Millán, Oliver Tonet, Federico Carpi, Michael Broschart, Pierre Ferrez, et. all, Prospects of brain-machine interfaces for space system control, Acta Astronautica, 64(4): 448-456, 2009

[4] Elisabeth V.C. Friedrich, Dennis J. McFarlanda, Christa Neuperb, Theresa M. Vaughan, Peter Brunner, Jonathan R. Wolpaw, A scanning protocol for a sensorimotor rhythm-based brain-computer interface, Biological Psychology, pp. 80(2): 169-175, 2009

[5] Marcel van Gerven, Ole Jensen, Attention modulations of posterior alpha as a control signal for two-dimensional brain-computer interfaces, Journal of Neuroscience Methods, 179(1): 7884

[6] Lucia Rita Quitadamo, Maria Grazia Marciani, Gian Carlo Cardarilli, Luigi Bianchi, Describing Different Brain Computer Interface Systems Through a Unique Model: A UML Implementation, Neuroinform, 6: 81-96, 2008, DOI 10.1007/s12021-008-9015-0

[7] I.H. Iversen, N. Ghanayim, A. Kübler, N. Neumann, N. Birbaumer, J. Kaiser, A braincomputer interface tool to assess cognitive functions in completely paralyzed patients with amyotrophic lateral sclerosis, Clinical Neurophysiology, 119(10): 2214-2223, 2008

[8] G. Pfurtscheller, T. Solis-Escalante, Could the beta rebound in the EEG be suitable to realize a "brain switch"? , Clinical Neurophysiology, 120(11): 24-29, 2008 
[9] Marios G. Philiastides, Paul Sajda, EEG-Informed fMRI Reveals Spatiotemporal Characteristics of Perceptual Decision Making, The Journal of Neuroscience, 27(48):13082-13091, 2007

[10] A. Vuckovic, F. Sepulveda, Quantification and visualisation of differences between two motor tasks based on energy density maps for brain-computer interface applications, Clinical Neurophysiology, 119(2): 446-458, 2008

[11] Oliver Tonet, Martina Marinelli, Luca Citi, Paolo Maria Rossini, Luca Rossini, Giuseppe Megali, et. all, Defining brain -machine interface applications by matching interface performance with device requirements, Journal of Neuroscience Methods, Brain-Computer Interfaces (BCIs), 167(1): 91-104, 2008

[12] Hyun K. Kim, Shinsuk Park, Mandayam A. Srinivasan, Developments in brain-machine interfaces from the perspective of robotics, Human Movement Science, 28(2): 191-203, 2009

[13] Febo Cincotti, Donatella Mattia, Fabio Aloise, Simona Bufalari, Gerwin Schalk, Giuseppe Oriolo, et. all, Non-invasive brain-computer interface system: Towards its application as assistive technology, Brain Research Bulletin, Special Issue: Robotics and Neuroscience, 775(6): 796-803, 2008

[14] F. Galán, M. Nuttin, E. Lew, P.W. Ferrez, G. Vanacker, J. Philips, J. del R. Millán, A brain-actuated wheelchair: Asynchronous and non-invasive Brain-computer interfaces for continuous control of robots, Clinical Neurophysiology, 119(9): 2159-2169, 2008

[15] Chia-Lin Chang, Zhanpeng Jin, Hou-Cheng Chang, Allen C. Cheng, From neuromuscular activation to end-point locomotion: An artificial neural network-based technique for neural prostheses, Journal of Biomechanics, 42(8): 982-988, 2009

[16] Febo Cincotti, Donatella Mattia, Fabio Aloise, Simona Bufalari, Laura Astolfi, Fabrizio De Vico Fallani, et. all, High-resolution EEG techniques for brain-computer interface applications, Journal of Neuroscience Methods, Brain-Computer Interfaces (BCIs), 167(1): 31-42, 2008

[17] Valerie Morash, Ou Bai, Stephen Furlani, Peter Lina, Mark Halletta, Classifying EEG signals preceding right hand, left hand, tongue, and right foot movements and motor imageries, Clinical Neurophysiology, 119(11): 2570-2578, 2008

[18] Vadim V. Nikulin, Friederike U. Hohlefeld, Arthur M. Jacobs, Gabriel Curio, Quasimovements: A novel motor-cognitive phenomenon, Neuropsychologia, 46(2): 727-742, 2008

[19] Farhad Faradji, Rabab K. Ward, Gary E. Birch, Plausibility Assessment of a 2-State SelfPaced Mental Task-Based BCI Using the No-Control Performance Analysis, Journal of Neuroscience Methods, 180(2): 330-339, 2009

[20] Daniel Pérez-Marcos, Jens-Uwe Knote, Reinhard Both, Galina Ivanova, Quantification of cognitive-induced brain activity: An efficient method for online applications, Computers in Biology and Medicine, 38(11-12): 1194-1202, 2008

[21] Seong-eun Ro, Joon Hwan Choi, Taejeong Kim, A new action potential classifier using 3Gaussian model fitting, Advances in Neural Information Processing (ICONIP 2006) / Brazilian Symposium on Neural Networks (SBRN 2006), Neurocomputing, 71(16-18): 3631-3634, 2008 
[22] Mingjun Zhong, Fabien Lotte, Mark Girolami, Anatole Lécuyer, Classifying EEG for brain computer interfaces using Gaussian processes, Pattern Recognition Letters, 29(3):354-359, 2008

[23] Jo-Anne Ting, Aaron D'Souza, Kenji Yamamoto, Toshinori Yoshioka, Donna Hoffman, Shinji Kakeif, et. all, Variational Bayesian least squares: An application to brain -machine interface data, Neural Networks, 21(8): 1112-1131, 2008

[24] Tonio Balla, Evariste Demandt, Isabella Mutschler, Eva Neitzel, Carsten Mehring, Klaus Vogt, et. all, Movement related activity in the high gamma range of the human EEG, NeuroImage, 41(2): 302-310, 2008

[25] Shang-Ming Zhou, John Q. Gan, Francisco Sepulveda, Classifying mental tasks based on features of higher-order statistics from EEG signals in brain-computer interface, Information Sciences, 178(6): 1629-1640, 2008

[26] F. Darvas, J.G. Ojemann, L.B. Sorensen, Bi-phase locking - a tool for probing non-linear interaction in the human brain, NeuroImage, 46(1): 123-132, 2009

[27] Wei-Yen Hsu, Yung-Nien Sun, EEG-based motor imagery analysis using weighted wavelet transform features, Journal of Neuroscience Methods, 175(2): 310-318, 2009

[28] Wu Ting, Yan Guo-zheng, Yang Bang-hua, Sun Hong, EEG feature extraction based on wavelet packet decomposition for brain computer interface, Measurement, 41(6): 618-62, 2008

[29] Dean Cvetkovic, Elif Derya Übeyli, Irena Cosic, Wavelet transform feature extraction from human PPG, ECG, and EEG signal responses to ELF PEMF exposures: A pilot study, Digital Signal Processing, 18(5): 861-874, 2008

[30] Vahid Abootalebi, Mohammad Hassan Moradib, Mohammad Ali Khalilzadeh, A new approach for EEG feature extraction in P300-based lie detection, Computer Methods and Programs in Biomedicine, 94(1): 48-57, 2009

[31] R. Murat Demirer, Mehmet Sirac Ozerdem, Coskun Bayrak, Classification of imaginary movements in ECoG with a hybrid approach based on multi-dimensional Hilbert-SVM solution, Journal of Neuroscience Methods, 178(1): 214-218, 2009

[32] W. A. Chaovalitwongse, P. M. Pardalos, On the time series support vector machine using dynamic time warping kernel for brain activity classification, Cybernetics and Systems Analysis, 44(1): 125-138, 2008

[33] Andreas Trřllund Boy, Ulrik Qvist Kristiansen, Martin Billinger, Omar Feix do Nascimento, Dario Farina, Identification of movement-related cortical potentials with optimized spatial filtering and principal component analysis, Biomedical Signal Processing and Control, 3(4): 300-304, 2008

[34] Gholamreza Salimi-Khorshidi, Ali Motie Nasrabadi, Mohammadreza Hashemi Golpayegani, Fusion of classic P300 detection methods' inferences in a framework of fuzzy labels, Artificial Intelligence in Medicine, 44(3): 247-259, 2008

[35] Vito Logar, Igor Škrjanc, Aleš Belič, Simon Brežan, Blaž Koritnik, Janez Zidar, Identification of the phase code in an EEG during gripping-force tasks: A possible alternative approach to the development of the brain-computer interfaces, Artificial Intelligence in Medicine, 44(1): 41-49, 2008 
[36] Dzitac S., Felea I., Dzitac I., Vesselenyi T., (2008), An Application of Neuro-Fuzzy Modelling to Prediction of some Incidence in an Electrical Energy Distribution Center, International Journal of Computers, Communications and Control, ISSN 1841-9836, 3(S):287-292, 2008

[37] Coyle D, Prasad G, McGinnity T.M., Faster self-organizing fuzzy neural network training and a hyperparameter analysis for a brain-computer interface, IEEE Trans Syst Man Cybern B Cybern., 39(6):1458-71, 2009

[38] Palaniappan R., Paramesran R., Nishida S., Saiwaki N., A new brain-computer interface design using fuzzy ARTMAP, IEEE Transactions on neural systems and rehabilitation engineering, ISSN 1534-4320, 10(3): 140-148, 2002

[39] Bang-hua Y., Guo-zheng Y., Ting W., Rong-guo Y., Subject-based feature extraction using fuzzy wavelet packet in brain-computer interfaces, Signal Processing, 87(7): 1569-1574, 2007

[40] Vesselenyi T., Dzitac I., Dzitac S., Hora C., Porumb C., Preliminary Issues On BrainMachine Contextual Communication Structure Development, Soft Computing Applications, 2009. SOFA '09. 3rd International Workshop on, ISBN 978-1-4244-5054-1, pp.37-42, IEEE Xplore DOI 10.1109/SOFA.2009.5254882, 2009

[41] Darvishi, S.; Al-Ani, A. Brain-Computer Interface Analysis using Continuous Wavelet Transform and Adaptive Neuro-Fuzzy Classifier, Engineering in Medicine and Biology Society, 2007. EMBS 2007. 29th Annual International Conference of the IEEE, 3220 - 3223.

[42] Pfurtscheller, G.: EEG event-related desynchronization (ERD) and event-related synchronization (ERS), In E. Niedermeyer, F.H. Lopes da Silva (eds.) Electroencephalography: basic principles, clinical applications and related fields, 4th edition, Williams and Wilkins, Baltimore, MD, 958-967, 1999

[43] Zadeh L. A., The Role of Fuzzy Logic in Modeling, Identification and Control, Modeling, Identification and Control, 15(3):191-203, 1994

[44] Zadeh L. A., Fuzzy Logic and the Calculus of Fuzzy If-Then Rules, Proceedings of SYNAPSE'91, Tokyo, Japan, 1991

[45] Zadeh L. A., Toward extended fuzzy logic-A first step, Fuzzy Sets and Systems, 160: 31753181,2009

[46] ***, MATLAB, User Guide, MathWorks, 2008 